\title{
Utilization of Compressed Sampling for PAPR Reduction in OFDM IEEE-802.11a System
}

\author{
Hussein Al-Moukhles, Member, IEEE
}

\begin{abstract}
Being spectrally efficient, easily implemented, and highly immune to selective channel imperfections and multipath fading, the Orthogonal Frequency Division Multiplexing (OFDM) can provide a sufficiently robust and high data rate modulation technique for emerging wired and wireless telecommunication applications. However, a major drawback of OFDM that is represented by its high Peak-to-Average Power Ratio (PAPR) of the transmitted signal, which leads to degrade the system's transmission accuracy. In this paper, a Compressed Sampling (CS) based approach is considered for reducing the PAPR without lowering its transmission capacity or affecting its Bit Error Rate (BER) performance. The proposed scheme adds a sampling stage after the IFFT block in the transmitter side, that is compressively represents the transmitted signal by fewer symbols that is transmitted instead of the original signal. At the receiver side, the received compressively sampled signal is then recovered before the FFT block by following the Basis Pursuit ( $\ell 1$-norm) algorithm. The proposed scheme shows an enhanced PAPR and BER performances while preserving the rest of the system performance aspects.
\end{abstract}

Index Terms - OFDM, Compressed Sampling, PAPR, Basis Pursuit.

\section{INTRODUCTION}

Due to its high immunity to selective fading channels during the high data transmission, the Orthogonal Frequency Division Multiplexing (OFDM) represents a promising approach for Multiple-Input Multiple-Output (MIMO) systems [1]. Under the same bandwidth, the MIMO-OFDM scheme can achieve both, a high performance and an efficient spectrum utilization [2], which make it possible for IEEE 802.11a, IEEE 802.16e, and 4G-LTE wireless networks to be implemented.

In the other side, the large value of the Peak-to-Average Power Ratio (PAPR) is a major drawback of MIMO-OFDM system. For some data constellations, the High-Power Amplifier (HPA) is forced to work in its characteristic's nonlinear region, which is distorting the OFDM signal, and resulted in an additional interference and a high Bit Error Rate (BER) degradations at the reception side [3].

Manuscript received February 11, 2021; revised July 20, 2021. Date of publication August 26, 2021. Date of current version August 26, 2021. The associate editor prof. Joško Radić has been coordinating the review of this manuscript and approved it for publication.

Hussein Al-Moukhles is with the University of Thi-Qar, College of Engineering, Electrical and Electronic Engineering Department (e-mail: Hussein-n@utq.edu.iq).

Digital Object Identifier (DOI): 10.24138/jcomss-2021-0033
Consequently, numerous PAPR reduction schemes have been proposed in the last decades [4]-[18]. In [4] Wang, S. H. et al. proposed a pre-coded OFDM scheme based on a perfect Gaussian integer sequence transform matrix that is both unitary and circulant which reduced complexity and PAPR artifacts with a less dynamic range compared to T-OFDM and typical OFDM. In [5], a hybrid companding technique based on precoding matrix and $\mathrm{Mu}$-law is proposed and resulted in BER performance and PAPR reduction trade-off. Coding techniques, like Arithmetic code and Reed-Solomon code, are utilized and compared for PAPR reduction in OFDM and F-OFDM modulation techniques in [6].

Based on Selected Mapping (SLM) strategy, Wang L. and Liu J. proposed in [7] a partial phase weighting SLM algorithm for PAPR reduction that reducing the complexity of the conventional SLM technique, which required a large number of IFFT stages and sending a Side Information (SI) to the receiver. In [8], a modified Hadamard-based SLM algorithm is proposed to overcome the transmitting of SI requirement in typical Hadamard-based PTS technique; which reduces the bandwidth efficiency. B. Lekouaghet et al. in [9] applied normalized Riemann matrix (RM) rows and a modified RM diagonal elements, along with rows of the symmetric Toeplitz matrix, as phase sequences for SLM strategy to reduce the PAPR in OFMD system.

Partial Transmit Sequence (PTS) is another effective scheme for PAPR reduction hat is partially transmitting the OFDM symbols as clusters with different phase rotations. T. Mata et al. in [10] proposed reducing the PTS complexity by utilizing the Artificial Bee Colony (ABC) algorithm. PTS is also modified in [11] by pre-combining the approximate gradient project for further PAPR reduction, with maintaining a high BER performance. A joint pre-distortion and PTS technique in [12] is proposed to extend the linear region of the HPA to maintain the BER performance. PTS and precoding techniques are combined in a hybrid scheme in [13] to reduce the number of candidate clusters and system calculations.

Duanmu, C. and Chen H. in [14] integrated SLM and PTS to utilize advantages of both techniques. The proposed hybrid SLM-PTS algorithm enhanced the PAPR and BER performances, and reducing the system complexity when compared with applying SLM or PTS separately.

A modified tone-reservation (TR) schemes based on signalto-clipping noise ratio (SCR) are proposed in [15]. Scaling SCR 
and Multiple Scaling SCR proposed techniques reduced the computational complexity and PAPR and improved the BER performance.

In [16], a PAPR and out-of-band (OOB) components reduction strategy is proposed based on Discrete Fourier Transform (DFT) and Windowing and Reconstruction (WR-) OFDM system. K. Liu et al. [17] proposed receiving method and PAPR and OOB radiations reduction algorithm based on Nonlinear Companding Transform (NCT).

An Artificial Neural Networks (ANN-) based algorithm is proposed in [18] to enhance BER and PAPR performances simultaneously. The proposed algorithm incorporated a multilayer feed forward ANN with Levenberg-Marquardt training algorithm. However, one effective criterion of reducing the PAPR is to ensure a high transmission (efficient bandwidth) and to maintain the overall OFDM system performance.

In this paper, a Compressed Sampling (CS) based PAPR reduction scheme is proposed for IEEE 802.11a standard MIMO-OFDM system. The proposed algorithm utilizes the property of sampling the compressible signal, and the corresponding recovery technique provided by CS to simultaneously reduce the PAPR and maintain the transmission performance of the OFDM system.

This paper is organized as follows: In section I, a general introduction to the MIMO-OFDM system and its importance is presented, and the PAPR artifact that degrades its performance is illustrated along with the most recent techniques to overcome it. In section II, the typical OFDM system block diagram and a mathematical description of the PAPR drawback are illustrated in detail. In section III, the CS theory, and sufficient conditions that make it applicable, are illustrated. In section IV, the block diagram of the proposed CS-based PAPR reduction algorithm is presented. In section $\mathrm{V}$, simulation results of the proposed algorithm for 802.11a standard specifications are presented, discussed, and compared to other PAPR reduction techniques. Section VI, concludes the paper.

\section{OFDM SYSTEM AND PAPR}

The block diagram of the typical OFDM system is shown in Fig. 1 [19]. The binary data stream at the input side is mapped into certain modulation scheme, and then converted to multiple low bit-rate parallel data streams. IFFT then successively transformed the parallel data streams to generate the orthogonal modulated data. The transformed data then converted into serial

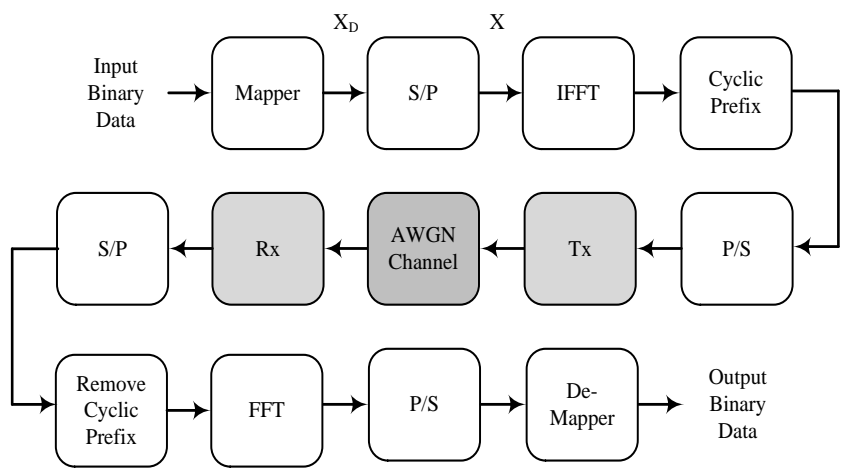

Fig. 1. The block diagram of the typical OFDM transmitter and receiver. form to generate the time domain OFDM signal and then transmitted through a HPA transmitter. An inverse procedure implemented to recover the data.

A mapped data block with length $N$ is

$$
\boldsymbol{X}_{\boldsymbol{D}}=\left[\begin{array}{llll}
X_{0} & X_{1} & \cdots & X_{N-1}
\end{array}\right]
$$

where $N$ is equal to the number of sub-carriers (data inputs of IFFT) and $\boldsymbol{X}=\left(\boldsymbol{X}_{\boldsymbol{D}}\right)^{\boldsymbol{T}}$. Each symbol $X_{n}(n=0,1, \ldots, N-1)$ with duration $T$ in the data block $\boldsymbol{X}$ used to modulate one subcarrier $e^{j 2 \pi f_{n} t}$ of the IFFT. The $N$ sub-carriers are orthogonal according to the IFFT properties, that is $f_{n}=n \Delta f$, where $\Delta f=$ $1 / N T$ and $N T$ is the duration of an OFDM data block. The complex envelope of the transmitted OFDM is given by [24]

$$
x(t)=\frac{1}{\sqrt{N}} \sum_{n=0}^{N-1} X_{n} e^{j 2 \pi f_{n} t}, 0 \leq t \leq N T
$$

which is represented as $N$ harmonically related exponentials. For some data blocks, all these harmonics (or sub-carriers) are added at their maximum points and hence a high PAPR is appeared. The PAPR for the transmitted signal can be calculated as [24]

$$
P A P R=\frac{\max \left\{|x(t)|^{2}\right\}}{E\left\{|x(t)|^{2}\right\}}
$$

where the numerator is the maximum power of the time domain OFDM signal and the denominator is its expectation. For an OFDM signal with $\mathrm{N}$ subcarriers, the maximum PAPR is

$$
P A P R_{\text {max }}=10 \log _{10}(N)
$$

\section{COMPRESSED SAMPLING}

Losing the information; according to Shannon/Nyquist's sampling theorem; can be avoided by capturing a signal at a sampling rate that is at least two times faster than the sampled signal's bandwidth [20]. When the transmission bandwidth in digital communication, storage capacity including video and image capturing devices, or the analogue-to-digital conversion speed in imaging systems such as radars and medical instruments, represent a limitation, then the Shannon /Nyquist's sampling rate represents an expensive choice.

Alternatively, other sampling rate is proposed by Donoho [21]; named compressed sampling (CS); for capturing and representing compressible signals - which are the case of most signals in the nature - by lower sampling rate than and by fewer sample that are required by Shannon/Nyquist theorem. The main idea of CS is to linearly project the signal in a space with lower number of basis than the original signal's space, then incorporating optimization processes to reconstruct the original signal [21], [22].

Considering the $N \times 1$ column vector $\boldsymbol{x} \in \mathbb{R}^{N}$ with elements $x[n](n=1,2, \ldots, N)$ as a one-dimensional discrete signal with 
finite-length $N$, then it is can be represented by $N$ vector orthonormal basis $\left\{\boldsymbol{\psi}_{\boldsymbol{i}}\right\}_{i=1}^{N}$ as

$$
\boldsymbol{x}=\sum_{i=1}^{N} \boldsymbol{s}_{i} \boldsymbol{\psi}_{i}
$$

or, $\boldsymbol{x}=\boldsymbol{\Psi} \boldsymbol{s}$, where $\boldsymbol{\Psi}$ is an $N \times N$ basis matrix $(\boldsymbol{\Psi}=$ $\left.\left[\boldsymbol{\psi}_{1}\left|\boldsymbol{\psi}_{2}\right| \ldots \mid \boldsymbol{\psi}_{N}\right]\right)$ and $\boldsymbol{s}$ is the weighting coefficients $N \times 1$ column vector such that

$$
\boldsymbol{s}_{i}=\left\langle\boldsymbol{x}, \boldsymbol{\psi}_{i}\right\rangle=\boldsymbol{\psi}_{i}^{T} \boldsymbol{x}
$$

Obviously, the vector $\boldsymbol{s}$ is respected as the representation of the discrete signal $\boldsymbol{x}$ in the domain that spanned by $\boldsymbol{\Psi}$ basis.

If the vector $\boldsymbol{s}$ has only $k$ non-zero entries, then the signal $\boldsymbol{x}$ is $k$-sparse, that is, it can be represented as a linear combination of only $k$-basis in the $\boldsymbol{\Psi}$ domain. Additionally, if $\boldsymbol{s}$ has $(N-k)$ zero (or comparatively small) elements, then the discrete signal $\boldsymbol{x}$ is said to be a compressible signal.

Given that $\boldsymbol{x}$ have an acceptable degree of compressibility, its representation vector $\boldsymbol{s}$ can be directly acquired by CS technique without performing intermediate framework such as sample-then-compress in Transform Coding [23]. This acquiring process includes the computing of $M \times 1$ measurement vector $\boldsymbol{y}_{i}$; where $M<N$; by performing $M$ linear measurements through inner product among $\boldsymbol{s}$ and collecting vectors as $\boldsymbol{y}_{j}=\left\langle\boldsymbol{x}, \boldsymbol{\phi}_{j}\right\rangle=\boldsymbol{\phi}_{j}^{T} \boldsymbol{x}$, where $\left\{\boldsymbol{\phi}_{i}\right\}_{j=1}^{M}$. Accordingly, the rows $\boldsymbol{\phi}_{j}^{T}$ can be represented as $M \times N$ matrix $\boldsymbol{\Phi}$, and thus $\boldsymbol{y}=\boldsymbol{\Phi} \boldsymbol{x}$. Equation (5) can be re-written as

$$
y=\Phi x=\Phi \Psi s=\Theta s
$$

where $\Theta$ is an $M \times N$ matrix and it is independent of the original signal $\boldsymbol{x}$.

Maintaining the information of the $k$-sparse signal $x \in \mathbb{R}^{N}$ from damaging during the reduction of its dimensionality to $\boldsymbol{y} \in$ $\mathbb{R}^{M}$ by the measurement matrix $\boldsymbol{\Phi}$, and its recovering from only fewer measurements $M \approx k$, are that main two problems in CSbased system.

These two requirements can be made available by satisfying two important and sufficient conditions, the first is called Restricted Isometry Property (RIP) [22], and the second is the incoherency between $\boldsymbol{\Phi}$ and $\boldsymbol{\Psi}$. The RIP condition stated that for any arbitrary $k$-sparse vector $\boldsymbol{u}$, and for some $\epsilon>0, \Theta$ must be satisfies the following inequality [22]

$$
1-\epsilon \leq \frac{\|\Theta u\|_{2}^{2}}{\|\boldsymbol{u}\|_{2}^{2}} \leq 1+\epsilon
$$

while the incoherency condition required that $\mu(\boldsymbol{\Phi}, \boldsymbol{\Psi})$ to be relatively small where

$$
\mu(\boldsymbol{\Phi}, \boldsymbol{\Psi})=\sqrt{N} \max _{1 \leq i, j \leq N}\left|\left\langle\boldsymbol{\phi}_{i}, \boldsymbol{\psi}_{j}\right\rangle\right|
$$

is the correlation between $\boldsymbol{\Phi}$ and $\boldsymbol{\Psi}$ matrices.
For instance, selecting the elements of the measurement matrix $\boldsymbol{\Phi}$ to be Gaussian identically independent distributed (i.i.d.) with $m=0$ and $\sigma^{2}=1 / N$ insures a highly probable achievement of both RIP and incoherency requirements in all cases of the orthonormal basis matrix $\boldsymbol{\Psi}$ if the following condition is satisfied

$$
M \geq \operatorname{cKlog}\left(\frac{N}{K}\right) \ll N
$$

where $c$ is a small constant [21], [22], [24].

The reconstruction of the original signal $\boldsymbol{x}$, or its representative in the $\boldsymbol{\Psi}$ domain $\boldsymbol{s}$, requires the measurement vector $\boldsymbol{y} \in \mathbb{R}^{M}$ and $\boldsymbol{\Phi}$ and $\boldsymbol{\Psi}$ matrices, after satisfying the aforementioned necessities. Reconstructing algorithm based on solving the underdetermined system of linear equations in (7) which has infinitely many solutions since $M<N$ [25].

Solving (3) is based on finding the sparsest solution in $M-$ or $N$ - dimensional space to determine $\boldsymbol{s}$ or $\boldsymbol{x}$, respectively, with minimum $\ell p$-norm that can be defined for vector $\boldsymbol{u}$ with cardinality $N$ as $\|\boldsymbol{u}\|_{p}=\left(\sum_{i=1}^{N}\left|u_{i}\right|^{p}\right)^{1 / p}$.

In this paper, Basis Pursuit algorithm [26] that is based on $\ell 1-$ norm minimization problem solution is used for signal reconstruction.

\section{PROPOSED CS-BASED SYSTEM}

The proposed system is shown in Fig. 2. In the transmitter side, firstly the input bit-stream is mapped to BPSK, to fit the OFDM 802.11a standard, then reshaped to fit the input of the IFFT by a S/P conversion process. Rows of the matrix at the S/P output represent the input symbols of the IFFT while its columns represent the total transmitted symbols.

The reshaped BPSK bit-stream is then applied to the IFFT input, and at the IFFT output, the CS block reduces the number of transmitted symbol through compressively sampling the resulted matrix columns by a ratio of 1-to-10 using the mechanism of (7). It is then paralleled by a $25 \%$ cyclic prefix. The new matrix is converted back to serial through a P/S stage, and transmitted through a HPA to the receiving side.

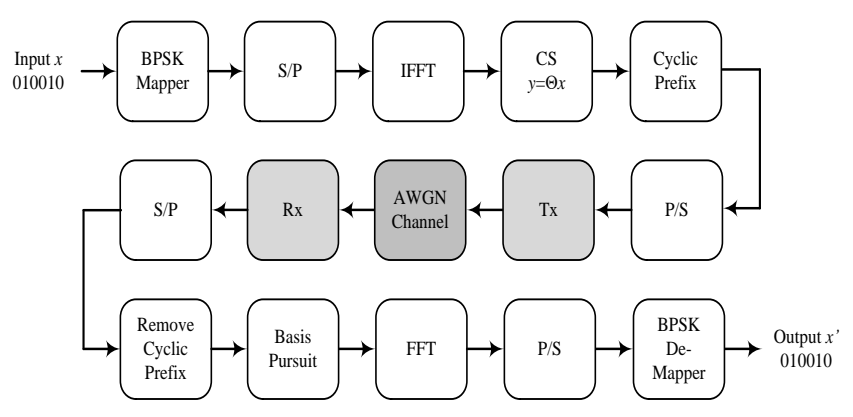

Fig. 2. The Proposed CS-Based OFDM 802.11a Model.

The channel is assumed to add Additive White Gaussian Noise (AWGN) to the transmitted OFDM signal. At the receiver side; and after its reception; the serial signal is converted to parallel and then to a removing cyclic prefix block. The resulted 
reduced matrix is then reconstructed by following a Basis Pursuit recovering algorithm though $\ell 1$-norm minimization to restore the compressively sampled symbols matrix to its original size before it is inputted to an FFT stage. Finally, the parallel BPSK data is converted to serial and de-mapped to output the bit-stream of the transmitted signal.

The measurement matrix $\boldsymbol{\Phi}$ and the representation basis matrix $\boldsymbol{\Psi}$ are selected to be a Gaussian i.i.d. random matrix with $m=0$ and $\sigma^{2}=1 / N$ and a Discrete Cosine Transform (DCT) matrix, respectively. The same matrices are stored at the transmitter and receiver sides and hence, no side information is required to transmit them between the transmitting and receiving terminals.

If $N$ is the number of bits to be transmitted, then the output from the BPSK mapper is

$$
\boldsymbol{x}_{\text {BPSK }}=\left[\begin{array}{llllll}
+1 & -1 & -1 & +1 & \ldots & -1
\end{array}\right]_{1 \times N}
$$

This BPSK signal is converted from serial to parallel by the $\mathrm{S} / \mathrm{P}$ converter to be reshaped as

$$
\boldsymbol{x}_{\text {Freq }}=\left[\begin{array}{ccc}
x_{\text {Freq }}(1,1) & \ldots & x_{\text {Freq }}\left(1, \frac{N}{M}\right) \\
\vdots & \ddots & \vdots \\
x_{\text {Freq }}(M, 1) & \ldots & x_{\text {Freq }}\left(M, \frac{N}{M}\right)
\end{array}\right]_{M \times\left(\frac{N}{M}\right)}
$$

After the IFFT transformation, $\boldsymbol{x}_{\boldsymbol{F r e q}}$ is transformed row by row to the time-domain parallel signal $\boldsymbol{x}_{\text {Time }}$ that is equal to

$$
\boldsymbol{x}_{\text {Time }}=\left[\begin{array}{ccc}
x_{\text {Time }}(1,1) & \ldots & x_{\text {Time }_{\left(1, \frac{N}{M}\right)}} \\
\vdots & \ddots & \vdots \\
x_{\text {Time }_{(M, 1)}} & \ldots & x_{\text {Time } \left._{(M,}, \frac{N}{M}\right)}
\end{array}\right]_{M \times\left(\frac{N}{M}\right)}
$$

The resulted time-domain signal if then reduced compressively by CS block to $\boldsymbol{x}_{\text {Time }} \boldsymbol{e}^{\text {that is equal to }}$

$$
\boldsymbol{x}_{\text {Time }}{ }^{\prime}=\left[\begin{array}{ccc}
x_{\text {Time }_{(1,1)}} & \ldots & x_{\text {Time }_{\left(1, \frac{N}{M^{\prime}}\right)}} \\
\vdots & \ddots & \vdots \\
x_{\text {Time }_{\left(M^{\prime}, 1\right)}} & \ldots & x_{\text {Time }}\left(M^{\prime}, \frac{N}{M^{\prime}}\right)
\end{array}\right]_{M^{\prime} \times\left(\frac{N}{M^{\prime}}\right)}
$$

where $M^{\prime}<M . \boldsymbol{x}_{\text {Time }}{ }^{\prime}$ then transmitted serially through the channel. The reverse sequence of processes is followed in the receiver side and a Basis Pursuit recovering algorithm is placed before the FFT stage based on $\ell 1$-norm minimization problem to recover the original dimension of the BPSK in (12) before it is compressively sampled.

According to equation (4), it is clear that the maximum PAPR is forwardly proportional to $N$ and hence, it can be reduced by lowering the value of $N$. After the IFFT stage, the mechanism of CS reduced the number of the total transmitted symbols by a ratio of 1-to-10 as mentioned before, which leads to the reduction on $N$ prior to the transmission stage without effecting the transmission speed. At the receiver side, the complete output of the IFFT before sampling can be reconstructed by the Basis Pursuit block.

The computational resource and time consumed in the Basis Pursuit recovering algorithm is high when compared to other PAPR reduction algorithms. However, the other PAPR procedures, including signal scrambling, coding, clipping, ... etc., are either required a side information or distort the signal and lowering the BER performance.

\section{Simulation Results}

The proposed system is simulated using Matlab code based on OFDM 802.11a specifications such as FFT and IFFT input/output size $N=64$, number of subcarriers $=52$, and number of bit-per-symbol $=52$, with number of symbols $=$ 2500. The performance of the proposed model is simulated under $0 \sim 10$ bit-to-noise ratio $\left(E_{b} / N_{o}\right)$ environments assuming AWGN channel. The system performance's is also compared with typical OFDM, Discrete Cosine Transform (DCT) based OFDM, and clipping based OFDM systems. The simulation results can be extended to include OFDM signals with highorder constellations such as QPSK-OFDM or QAM-OFDM based OFDM systems.

In Fig.3, the PAPR reduction performance is compared for the proposed scheme with typical, DCT-based, and Clippingbased OFDM systems. The comparison is based on the calculation of the Complimentary Cumulative Distribution Function (CCDF) of the aforementioned different techniques. It is clear that the proposed CS-OFDM has outperforms BPSKBased typical OFDM, OFDM-DCT, and OFDM-Clipping Systems schemes in keeping the PAPR under a specified limit.

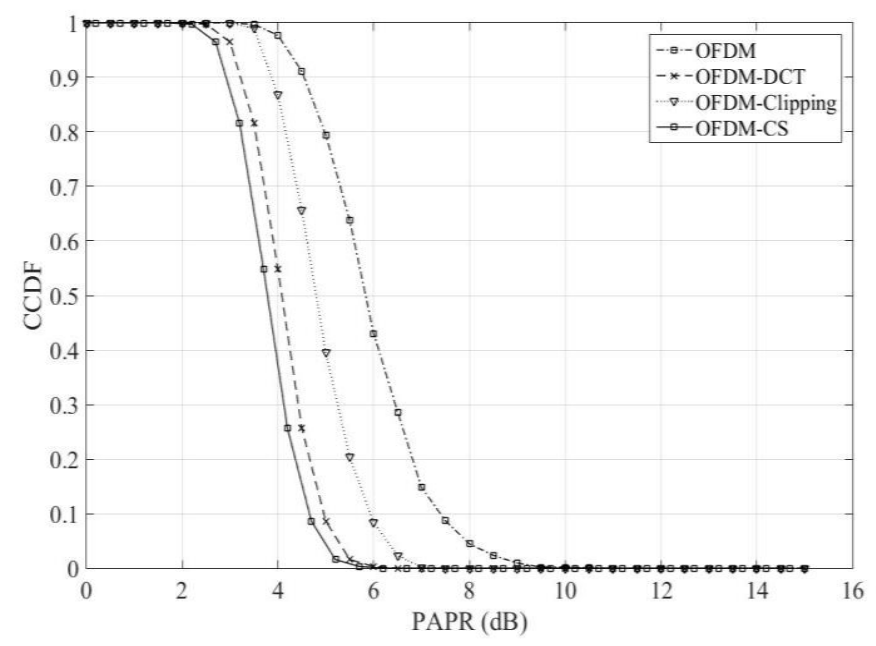

Fig. 3. PAPR-CCDF for BPSK-Based OFDM, OFDM-DCT, OFDMClipping, and OFDM-CS Systems.

Fig. 4 shows the symbols normalized power for OFDM, OFDM-DCT, OFDM-Clipping, and OFDM-CS Systems. A comparison of number of peaks and their heights among the different systems shows a better (lower) variance for CS- 
OFDM system than other systems, since the peaks are clearly having a close value to the average value of the symbols normalized power. The last result comes as a result of random sampling of the original data matrix which is resulted in signal scrambling.

Finally, the BER performance is calculated and compared for theoretical, typical OFDM, OFDM-DCT, OFDM-Clipping, and OFDM-CS techniques as shown in Fig. 5. This performance is calculated for a range of bit-to-noise ratio $\left(E_{b} / N_{o}\right)$ of $0 \sim 10$ with AWGN channel. The simulation results clear that the CSOFDM scheme has a betted BER rate performance than the other systems for $\left(E_{b} / N_{o}\right)$ values of $0 \sim 7.5$ and then CS-OFDM start performing the same way of the other systems.

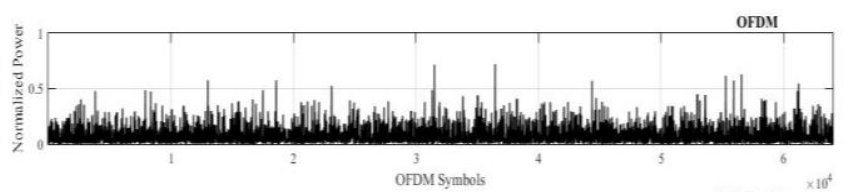

(a)

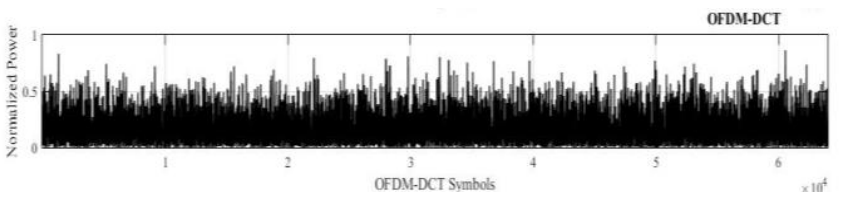

(b)

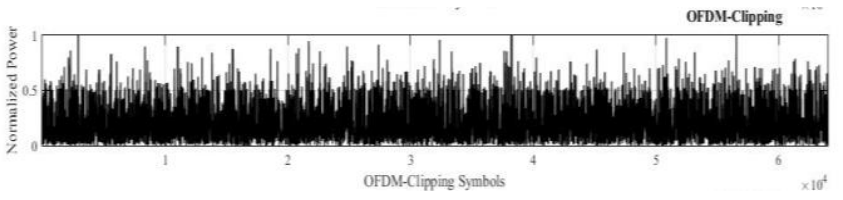

(c)

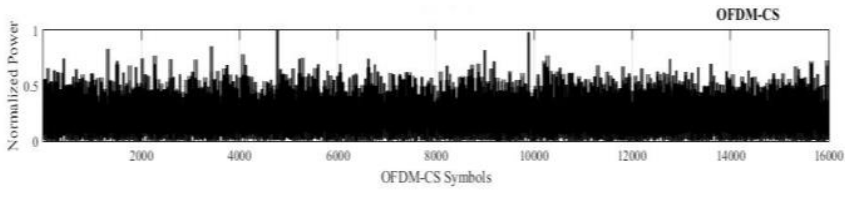

(d)

Fig. 4. Normalized Power for (a) OFDM, (b) OFDM-DCT, (c) OFDMClipping, and (d) OFDM-CS Systems.

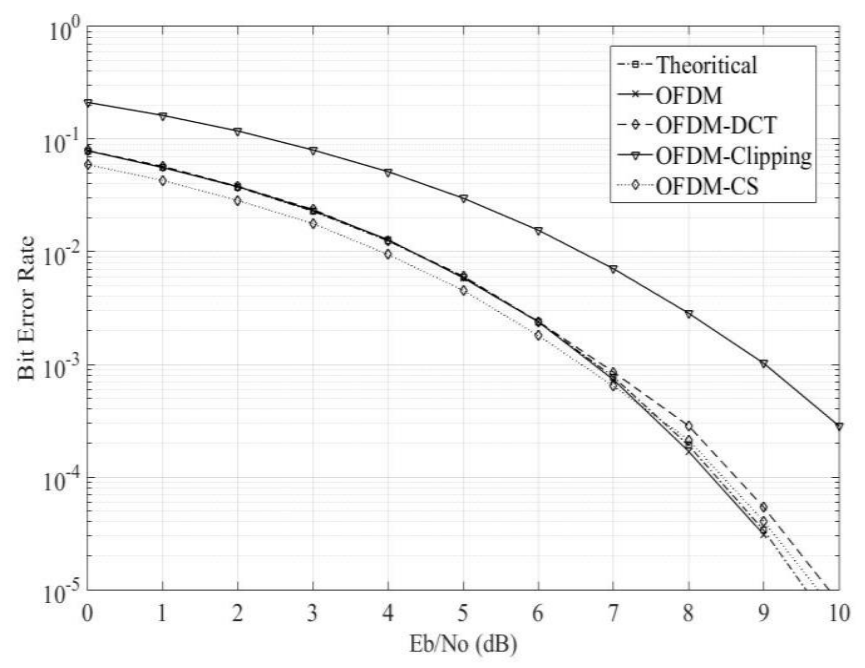

Fig. 5. BER with BPSK for Theoretical, Typical OFDM, OFDM-DCT,

\section{OFDM-Clipping, and OFDM-CS Techniques.}

\section{CONCLUDING REMARKS}

A CS-Based algorithm has been proposed to reduce the value of PAPR in OFDM system. The proposed system considered a compressive sampling of the original data matrix in the transmitter side before the IFFT stage, as well as recovering the original data by Basis Pursuit based algorithm stage in the receiver side after the FFT block. The proposed system enhanced the PAPR performance compared with typical, DCT, and Clipping-based OFDM schemes. The PAPR reduction is achieved without lowering the capacity of transmission or affecting its Bit Error Rate (BER).

\section{REFERENCES}

[1] Soo, Y., Kim, J., Yang, W. Y., \& Kang, C. G. (2010) "MIMO OFDM wireless communications with Matlab," London: Wiley.

[2] Urosevic, U., Veljovic, Z., \& Pejanovic-Djurisic, M. (2013) "MIMO solution for performance improvements of OFDM-CDMA system with pilot tone," Wireless Networks. doi:10.1007/s11276-013-0586-9.

[3] Ho-Lung H., (2017) "Application firefly algorithm for peak-to-average power ratio reduction in OFDM systems," Telecommun Syst, Vol. 65, pp.1-8. doi:10.1007/s11235-016-0208-9.

[4] Wang, S.-H., Li, C.-P., Lee, K.-C., \& Hsuan-Jung, S. (2015) "A novel low-complexity pre-coded OFDM system with reduced PAPR," IEEE Transactions on Signal Processing, Vol. 63, pp. 1366-1376.

[5] N. Sharan, S. K. Ghorai and A. Kumar, "Peak-to-Average Power Ratio (PAPR) Reduction Using Combination of Precoding and Companding Techniques for VLC OFDM Systems," 2019 TEQIP III Sponsored International Conference on Microwave Integrated Circuits, Photonics and Wireless Networks (IMICPW), Tiruchirappalli, India, 2019, pp. 149153, doi: 10.1109/IMICPW.2019.8933194.

[6] A. Idris, N. L. Mohd Sapari, M. Syarhan Idris, S. S. Sarnin, W. Norsyafizan Wan Mohamad and N. F. Naim, "Reduction of PAPR Using Block Coding Method and APSK Modulation Techniques for F-OFDM in 5G System," TENCON 2018 - 2018 IEEE Region 10 Conference, Jeju, Korea (South), 2018, pp. 2456-2460, doi: 10.1109/TENCON. 2018.8650304.

[7] Wang, L., \& Liu, J. (2015) "Partial phase weighting selected mapping scheme for peak-to-average power ratio reduction in orthogonal frequency division multiplexing system," IET Communications, Vol. 9, pp. 147-155.

[8] N. Li, M. Li and Z. Deng, "A modified Hadamard based SLM without side information for PAPR reduction in OFDM systems," in China Communications, vol. 16, no. 12, pp. 124-131, Dec. 2019, doi: 10.23919/JCC.2019.12.009.

[9] B. Lekouaghet, Y. Himeur and A. Boukabou, "Improved SLM technique with a new phase factor for PAPR reduction over OFDM signals," $0201 s t$ International Conference on Communications, Control Systems and Signal Processing (CCSSP), EL OUED, Algeria, 2020, pp. 8-12, doi: 10.1109/CCSSP49278.2020.9151592.

[10] T. Mata, P. Boonsrimuang and P. Boontra, "A PAPR Reduction Scheme based on Improved PTS with ABC Algorithm for OFDM Signal," 2018 15th International Conference on Electrical Engineering/Electronics, Computer, Telecommunications and Information Technology (ECTICON), Chiang Rai, Thailand, 2018, pp. 469-472, doi: 10.1109/ECTICon. 2018.8619887.

[11] Pachori, K., \& Mishra, A. (2015) "PAPR Reduction in MIMO-OFDM by using active partial sequence," Circuits, Systems, and Signal Processing. doi:10.1007/s00034-015-0039-z.

[12] Y. Moegiharto, A. M. Kautsar Bebyrahma and I. Anisah, "BER Performance of Joint PTS PAPR Reduction Technique and Wiener HPA Predistortion in OFDM System," 2019 International Electronics Symposium (IES), Surabaya, Indonesia, 2019, pp. 480-484, doi: 10.1109/ELECSYM.2019.8901566.

[13] R. Iwasaki and K. Ohuchi, "PAPR Reduction in OFDM Signal by Combining Partial Transmit Sequences with Precoding Matrix," 2018 12th International Conference on Signal Processing and Communication 
Systems (ICSPCS), Cairns, Australia, 2018, pp. 1-6, doi: 10.1109/ICSPCS.2018.8631723.

[14] Duanmu, C., \& Chen, H. (2014) "Reduction of the PAPR in OFDM systems by intelligently applying both PTS and SLM Algorithms," Wireless Personal Communication. doi:10.1007/s11277-013-1325-3.

[15] J. Wang, X. Lv and W. Wu, "SCR-Based Tone Reservation Schemes with Fast Convergence for PAPR Reduction in OFDM System," in IEEE Wireless Communications Letters, vol. 8, no. 2, pp. 624-627, April 2019, doi: 10.1109/LWC.2018.2890596.

[16] M. N. Hossain, T. Shimamura, D. Kim and H. Ryu, "Waveform Design of DFT-Spread WR-OFDM System for the OOB and PAPR Reduction," 2018 International Conference on Information and Communication Technology Convergence (ICTC), Jeju, 2018, pp. 792-796, doi: 10.1109/ICTC.2018.8539398.

[17] K. Liu, L. Wang and Y. Liu, "A new nonlinear companding algorithm based on tangent linearization processing for PAPR reduction in OFDM systems," in China Communications, vol. 17, no. 8, pp. 133-146, Aug. 2020, doi: 10.23919/JCC.2020.08.011.

[18] Mishra, A., Saxena, R., \& Patidar, M. (2014) "OFDM link with a better performance using artificial neural network," Wireless Personal Communications. doi:10.1007/s11277-013-593-y.

[19] N. K. Jadav, "A Survey on OFDM Interference Challenge to improve its BER," 2018 Second International Conference on Electronics, Communication and Aerospace Technology (ICECA), Coimbatore, 2018, pp. 1052-1058. doi: 10.1109/ICECA.2018.8474748.

[20] J. G. Proakis \& D. G. Manolakis, (1996 ) Digital Signal Processing Principles, Algorithms, and Applications, Prentice-Hall International Inc.

[21] D. Donoho (2006) "Compressed Sensing" IEEE Trans. Inform. Theory, Vol. 52, no. 4, pp. 1289-1306.

[22] E. Candès, J. Romberg \& T. Tao (2006) "Robust uncertainty principles Exact signal reconstruction from highly incomplete frequency information" IEEE Trans. Inform. Theory, Vol. 52, no. 2, pp. 489-509.

[23] R.G. Baraniuk, M. Davenport, R. DeVore, \& M.B. Wakin (2007) “A simple proof of the restricted isometry principle for random matrices", Constructive Approximation. Available: http://dsp.rice.edu/cs/jlcsv03.pdf

[24] R. G. Baraniuk, (2007) "Compressive Sensing [Lecture Notes]," IEEE Signal Processing Magazine, Vol. 24, no. 4, pp. 118-121. doi: 10.1109/MSP.2007.4286571.

[25] D. Donoho, H. Kakavand \& J. Mammen (2006) "The Simplest Solution to an Underdetermined System of Linear Equations," IEEE International Symposium on Information Theory, Seattle, WA.

[26] Z. M. Hussain, P. O'Shea, \& A. Z. Sadik (2011), "Digital Signal Processing”, Springer.

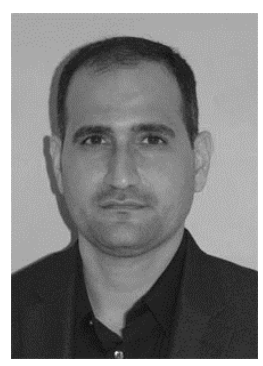

Hussein Al-Moukhles was born in State of Kuwait in Oct., $7^{\text {th }}, 1975$. He received the B.S. and M.S. degrees in electrical engineering from the University of Basra, Basra governorate, Iraq, in 1999 and 2002 respectively. From 2003 to 2007, he was an assistant lecturer at the electrical and electronic engineering department in university of Thi-Qar, Thi-Qar governorate, Iraq. Since 2008, he was a lecturer at the same department where he taught many subjects in electrical circuits analysis, logic design, electrical machines theory, mathematics, advanced mathematics, and digital signal processing. He is an IEEE member since 2016. Recently he received the Ph.D. degree in electrical and computers engineering from the Department of Electrical and Computer Engineering, Western Michigan University, Kalamazoo, MI, USA, in 2018. His $\mathrm{Ph}$.D. dissertation topic is WLAN-RSSI fingerprinting for Indoor positioning systems. His research interests include signal processing for communication and biomedical applications, digital image processing, and heterojunctions. 